Editorial

\title{
Do We Need Activity Scores or Simply Clear Criteria for Intestinal Ultrasound in Ulcerative Colitis?
}

\section{Torsten Kucharzik ${ }^{a}$, Christian Maaser ${ }^{b}$, Giovanni Maconi ${ }^{c}$}

\author{
${ }^{a}$ Klinikum Lüneburg, Department of Gastroenterology, Lüneburg, Germany ${ }^{b}$ Klinikum Lüneburg, IBD Outpatient \\ Department, Lüneburg, Germany ' $\mathrm{L}$ Sacco University Hospital, Department of Gastroenterology, Milan, Italy \\ *Corresponding author: Torsten Kucharzik, MD, PhD, Department of Gastroenterology, Lüneburg Hospital, Bögelstr. 1, \\ Lüneburg, Germany. Tel: +49 4131 772241; Fax: +49 4131 772245; Email: Torsten.Kucharzik@klinkum-lueneburg.de
}

Non-invasive diagnostic tools are playing increasingly relevant roles in monitoring inflammatory bowel diseases. Increasing data support the view that intestinal ultrasound [IUS] can be used as a reliable tool to determine and to monitor Crohn's disease activity. ${ }^{1-3}$ Consequently, the use of IUS to follow up active Crohn's disease is recommended in recent ECCO diagnostic guidelines. ${ }^{4}$ The role of IUS in ulcerative colitis [UC] is less well defined and fewer studies have determined its role in measuring disease activity. ${ }^{5-8}$

The study by M. Allocca et al. published in the current issue of JCC aims to develop an IUS score to assess disease activity and severity of UC using endoscopy as a comparator.

The key conclusions of the study are that colonic wall thickness $[\mathrm{CWT}]$ and vascularity of the colonic walls, namely colonic wall flow $[\mathrm{CWF}]$ as assessed by colour Doppler, were the most important features in assessing disease activity. In particular, the study showed that CWT > $3 \mathrm{~mm}$ coupled with CWF, or CWT as a single parameter, but with a cut-off of $>4.43 \mathrm{~mm}$, indicates disease activity with Mayo sub-score of 2-3, with sensitivity of 0.71 and specificity of 1.00. The single parameter of CWT $>3 \mathrm{~mm}$ gave better accuracy [0.88] with greatest sensitivity [0.89] and specificity [0.87]. Of Note, simple sonographic criteria (Humanitas ultrasonographic criteria [HUC]) showed high interobserver agreement with a kappa of 0.86 . Receiver operating characteristic curve analysis identified a score $\geq 6.3$ as an indicator of endoscopic activity [Mayo sub-score $\geq 2$ ], with a specificity of $100 \%$. When the authors combine the HUC with faecal calprotectin [FC], the accuracy of HUC did not change significantly, suggesting that ultrasound parameters alone are sufficient to determine endoscopic activity in UC.

This well-described trial underlines the role of IUS in determining disease activity in UC. It also strengthens the relevance of standardization of measurement of IUS findings as well as the requirement of developing IUS criteria for measuring disease activity in UC. Developing simple, statistically derived scores to determine disease activity as demonstrated in the current pilot study is a crucial step to standardize monitoring of the disease.

However, although these criteria are very simple and have an overall high specificity, they may miss the diagnosis of active inflammation in as many as three of ten patients, but are well in keeping with data in the literature. This result is expected given the typical mucosal damage associated with UC, but offers us the possibility to choose the best IUS criterion for individual patients. Among the criteria shown in the study, however, it appears that simply bowel wall thickening may provide the best performance in terms of sensitivity and specificity. However, the additional use of vascularization assessed with colour Doppler, or of inflammation by using FC, may increase the specificity or, conversely, the sensitivity, to exclude or confirm active inflammation.

The study also confirms the ease of detecting activity of UC by IUS, given that it may be simply revealed by an abnormal colonic wall thickening [> $3 \mathrm{~mm}$ ]. In fact, it is well known from the literature data, recently summarized in the European Guidelines for Ultrasound in IBD, that bowel wall thickening $>3 \mathrm{~mm}$ is the cut-off to assess the presence of IBD, and active UC in particular, ${ }^{9}$ and that the detection of abnormal wall thickening [>3 mm] is one of the most reproducible IUS parameters. ${ }^{10}$

Our own unpublished data suggest that measurement of CWT alone might be sufficient if CWT is determined in a standardized manner.

Although the current study could not show an additional effect of measuring FC, it is conceivable that a combination of IUS with FC may increase the accuracy in determining disease activity at least in a subgroup of UC patients. Measurement of FC may in particular increase sensitivity in patients with active rectal inflammation, where IUS shows low sensitivity. Unfortunately, patients with UC limited to the rectum and distal sigmoid colon were excluded in Allocca's study and therefore the accuracy reported in assessing activity in this study may not exactly reflect accuracy in the general UC population. Reduced sensitivity in patients with active disease limited to the mucosa without increased bowel wall thickening should also be taken into account. These false negatives harbour the risk of oversimplification with use of a score.

FC could therefore represent a valid tool in combination with IUS in general practice, but this remains to be demonstrated in future studies. Multicentre studies are therefore required to validate the HUC in larger cohorts and to evaluate its usefulness in monitoring UC. In particular, it will be mandatory to perform more studies investigating the value of the different IUS parameters that are required to determine disease activity as well as studies that evaluate the role of IUS in measuring and monitoring disease activity in UC. 
It is unclear how many parameters are required to develop a simple and reliable score for monitoring disease activity. It remains also to be defined if we need a score that can simply discriminate active vs non-active disease, or another one that can provide different degrees of disease activity, such as currently offered by endoscopic evaluation, which for this reason remains and will probably remain the reference standard for assessing UC activity.

However, preliminary data from a large multicentre trial in patients with active UC, whose disease activity has been monitored by using IUS, showed promising results, revealing changes in bowel wall thickness by IUS as early as 2 weeks after treatment initiation. ${ }^{11}$ With more robust data showing the utility of IUS in monitoring disease activity in IBD, IUS may become a cost-effective, safe and easy to use diagnostic tool that might replace or at least reduce the number of invasive colonoscopies for monitoring disease activity.

\section{Funding}

There has been no specific funding for this work.

\section{Conflict of Interest}

TK received honorary fees from Abbvie, Biogen, Boehringer Ingelheim, Ferring, Hospira, Mundipharma, Dr. Falk Pharma GmbH, Janssen, MSD Sharp \& Dome GmbH and Takeda Pharma GmbH. CM received honorary fees from Abbvie, Ferring, Dr. Falk Pharma GmbH, Janssen, MSD Sharp \& Dome GmbH and Takeda Pharma GmbH. GM received honorary fees from Abbvie, AlfaSigma, Allergan, Janssen-Cilag, Novartis and Takeda Pharma GmbH.

\section{Author Contributions}

TK, first draft of the editorial. CM, finalisation of the editorial including further relevant aspects. GM, second draft of the editorial including new relevant aspects.

\section{References}

1. Kucharzik T, Wittig BM, Helwig U, et al.; TRUST study group. Use of intestinal ultrasound to monitor Crohn's disease activity. Clin Gastroenterol Hepatol 2017;15:535-542.e2.

2. Bryant RV, Friedman AB, Wright EK, et al. Gastrointestinal ultrasound in inflammatory bowel disease: an underused resource with potential paradigm-changing application. Gut 2018;67:973-85.

3. Kucharzik T, Maaser C. Intestinal ultrasound and management of small bowel Crohn's disease. Therap Adv Gastroenterol 2018;11:1756284818771367.

4. Maaser C, Sturm A, Vavricka SR, et al. ECCO-ESGAR guideline for diagnostic assessment in inflammatory bowel disease. J Crohns Colitis 2018; Aug 23 (Epub ahead of print).

5. Maconi G, Ardizzone S, Parente F, Bianchi Porro G. Ultrasonography in the evaluation of extension, activity, and follow-up of ulcerative colitis. Scand J Gastroenterol 1999;34:1103-7.

6. Parente F, Greco S, Molteni M, et al. Role of early ultrasound in detecting inflammatory intestinal disorders and identifying their anatomical location within the bowel. Aliment Pharmacol Ther 2003;18:1009-16.

7. Parente F, Molteni M, Marino B, et al. Are colonoscopy and bowel ultrasound useful for assessing response to short-term therapy and predicting disease outcome of moderate-to-severe forms of ulcerative colitis?: a prospective study. Am J Gastroenterol 2010;105:1150-7.

8. Parente F, Molteni M, Marino B, et al. Bowel ultrasound and mucosal healing in ulcerative colitis. Dig Dis 2009;27:285-90.

9. Maconi G, Nylund K, Ripolles T, et al. EFSUMB Recommendations and Clinical Guidelines for Intestinal Ultrasound (GIUS) in Inflammatory Bowel Diseases. Ultraschall Med 2018;39:304-17.

10. Calabrese E, Kucharzik T, Maaser C, et al. Real-time interobserver agreement in bowel ultrasonography for diagnostic assessment in patients with Crohn's disease: an international multicenter study. Inflamm Bowel Dis 2018; doi: 10.1093/ibd/izy091

11. Maaser C, Petersen P, Helwig U, et al. Bowel ultrasound is useful in disease monitoring of ulcerative colitis patients: first analysis from the TRUST\&UC study in Germany. United Eur Gastroent J 2017; 5:P0369. 